\title{
Abstract Book
}

\section{1st ENSP Conference}

\section{on Tobacco Control}

\section{Research, Prevention and Treatment}

$$
\text { 5- } 7 \text { April } 2016
$$

Brussels, Belgium

Abstracts

\section{European Network}

for Smoking and Tobacco Prevention

Supported by

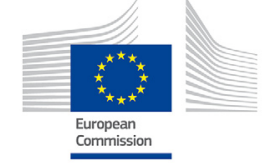




\section{FOREWORD}

\section{Welcome to the ENSP Tobacco Control Conference}

The European Network for Smoking and Tobacco Prevention (ENSP) has been active in tobacco control since 1997 and for the last 19 years has been playing an important role in setting and influencing policies at a European and international level. Spearheading ENSP activities and projects are national representatives, organisations, academics, doctors, lawyers, health care professionals, experts and activists who always worked together to fulfil the objectives and mission of ENSP.

ENSP is the only truly pan-European network active in tobacco control being the key grassroots organisation in implementing the World Health organization Framework Convention on Tobacco Control (WHO-FCTC) at a European level. This was acknowledged by the WHO-FCTC - Conference of Parties during the session in Uruguay (November 2010) when ENSP received FCTC observer status.

It is almost impossible to talk about FCTC in Europe without mentioning the involvement of at least one ENSP member. This united and collective effort is the essence, the heart and soul of the "Network" concept: each link makes the chain stronger; each node adds value to the structure. This added value is not only found in the strength of the partnership but also on a scientific level in everything ENSP does: its core activities, its EU projects but also its new scientific journal, "Tobacco Prevention \& Cessation".

Tobacco consumption remains the single largest avoidable cause of premature death in Europe, responsible for 700,000 deaths every year, and is the most significant cause of health inequalities. It is an interesting time for our work in tobacco control, as the world recognises that the most effective policies are those based on scientific studies and solid evidence.

Therefore, this year the conference is dedicated to three main topics:

RESEARCH - Research and science represent the base for lobby and advocacy activities. On 5th April, a special session is dedicated to the European research and Public Health Projects dealing with different tobacco control topics. In all those projects, ENSP is acting as a knowledge and networking hub, being a real support for researchers and partners involved in the projects' implementation.

PREVENTION - Next year ENSP celebrates 20 years of activities dedicated mainly to prevent tobacco consumption in Europe. During the conference, a series of workshops, plenary and parallel sessions aim to highlight its accomplishments based on constant guidance resulting from WHO FCTC and MPOWER strategies.

TREATMENT - Wednesday April 6th is fully dedicated to the WHO-FCTC Art. 14's implementation in Europe and we bring together, in a special training session, more than 50 experts from Armenia, Georgia, Romania, Russia and Ukraine, partner countries under the EPACTT Project developed in partnership with Global Bridges - Healthcare Alliance for Tobacco Dependence Treatment.

The above-mentioned topics demonstrate the diversity and complexity of our Network, which is dedicated to tobacco control in Europe. Furthermore, the International Society for the Prevention of Tobacco Induced Diseases has joined forces by sponsoring two symposiums on Thursday 7 April, reinforcing the importance of academic and scientific research in our work.

Bringing together more than 170 experts from over 30 countries, this Conference is a new step ahead in accomplishing our two top priority objectives as stated in our Statute:

- To have the WHO- Framework Convention on Tobacco Control implemented in Europe by 2020

- To reduce the prevalence of tobacco use in Europe to below 5\% by 2040.

During the conference, as you discover and meet special guests and speakers from the Academic and European Institutions, World Health Organization, national authorities and well known non-governmental organisations involved in tobacco control at regional, national and international level, we hope you have the chance to share your experiences and insights pertaining to various facets of tobacco control.

On behalf of ENSP members and Secretariat, we are honoured to invite you to our Tobacco Control Conference in Brussels, the capital city of Belgium and Europe, and would like to thank you, as well as all the presenters, without whom this event would not be possible, for your work, your dedication and your loyalty.

ENSP BOARD AND SECRETARIAT 


\section{Conference Proceeding}

\section{ABSTRACTS}

\section{ABSTRACT}

\section{Tobacco Products Directive - new opportunities for EU tobacco control}

Anna-Eva Ampelas ${ }^{1}$

'DG SANTE Unit B2, Belgium

The Tobacco Products Directive 2014/40/EU was adopted in 2014 and needs to be transposed by Member States by 20 May 2016. It sets out ambitious tobacco control measures in the areas of ingredients, packaging \& labelling, electronic cigarettes and tracking \& tracing. The new Directive focuses on preventing young people from taking up smoking. Tob. Prev. Cessation 2016;2(April Supplement):51 http://dx.doi.org/10.18332/tpc/62451

\section{ABSTRACT 2}

Calling on the European civil society to support global implementation of the WHO FCTC and its Protocol to Eliminate Illicit Trade in Tobacco Products

Vera Luiza da Costa e Silva

'WHO FCTC, Switzerland

The intervention of the Head of the Convention Secretariat will focus on how the European civil society could support global implementation of the WHO FCTC and its Protocol to Eliminate Illicit Trade in Tobacco Products. The Convention celebrated its 10 years of existence last February and currently has 180 Parties that committed themselves towards implementing its requirements. Almost all European states and the European Union have ratified the Convention and working on its implementation. The intervention will focus on the roles the European civil society could play to strengthen its contribution, at national, sub-regional and global levels, in implementation of the Convention. The Protocol to Eliminate Illicit Trade in Tobacco Products, a new international treaty, that will open new avenues for collaboration between the various sectors, is still to enter into force. Its implementation will also need support from the side of the civil society.

Tob. Prev. Cessation 2016;2(April Supplement):50

http://dx.doi.org/10.18332/tpc/62450

\section{ABSTRACT 3}

\section{Science for tobacco control policy}

Constantine Vardavas ${ }^{1}$
'Institute of Public Health of the American College of Greece, Greece

The recent adoption of the Tobacco Products Directive is a unique opportunity to enhance the regulation of tobacco products in the European Union. In this presentation a brief overview of the development of an EU common reporting format for submission of data on ingredients contained in tobacco and related products will be presented, as an example of European tobacco regulatory science.

Tob. Prev. Cessation 2016;2(April Supplement):49

http://dx.doi.org/10.18332/tpc/62449

\section{ABSTRACT 4}

Evaluating Tobacco Control Policies in 28 Countries (including 9 EU countries): The ITC Project

Geoffrey Fong

'University of Waterloo, Canada

Since its start in 2002, the ITC Project has been conducting evaluation studies of tobacco control policies via prospective cohort surveys of tobacco users in 28 countries, including 9 EU countries. This presentation will focus on the design of the ITC Project and how it differs from and complements existing evidence-gathering systems (monitoring and surveillance systems) in measuring and understanding the impact of FCTC policies. The presentation will also describe the ITC Project's most recent initiatives: (1) the EUREST-PLUS study focusing on measuring the impact of the Tobacco Products Directive, and (2) a large-scale international cohort study of e-cigarettes starting in the United States, Canada, England, and Australia. Tob. Prev. Cessation 2016;2(April Supplement):48 http://dx.doi.org/10.18332/tpc/62448

\section{ABSTRACT 5}

\section{Australian Tobacco Control Achievements}

Simon Chapman

' University of Sydney, Australia

With the historic implementation of plain tobacco packaging in December 2012, Australia has arguably the most comprehensive system of tobacco control policy and practice of any nation. Daily smoking prevalence in those aged 14+ was $12.8 \%$ (in 2013) with the next data set due later this 
year. Australia vies with Canada to have the lowest smoking prevalence of any nation. This presentation will outline the leading factors contributing to the decline, emphasising the population rather than individual/clinical focus of policies and programs; the interrelationship of distal and proximal factors in understanding the dynamics of the decline; and the vital role of media advocacy over decades that has conditioned public and political awareness and values, essential for fomenting policy change.

Tob. Prev. Cessation 2016;2(April Supplement):47 http://dx.doi.org/10.18332/tpc/62447

\section{ABSTRACT 6}

\section{Article 5.3 of the WHO Framework Tobacco Convention and the European Ombudsman's inquiry into tobacco lobbying}

Lambros Papadias'

'European Ombudsman (Belgium)

The Ombudsman investigates complaints about maladministration in the EU institutions, agencies and bodies. A complaint was brought by an NGO which claimed the Commission was not meeting its obligations under the WHO's Framework Convention on Tobacco Control. The Ombudsman agreed, finding that the Commission's approach to publicising meetings with tobacco lobbyists was, with the exception of DG Health, inadequate, unreliable and unsatisfactory. The Ombudsman was also concerned to find that certain meetings with lawyers representing the tobacco industry were not considered as meetings for the purpose of lobbying.

Tob. Prev. Cessation 2016;2(April Supplement):46

http://dx.doi.org/10.18332/tpc/62446

\section{ABSTRACT 7}

\section{Ingredients and additives in tobacco products \\ Martina Pötschke-Langer' \\ ${ }^{1}$ German Cancer Research Center, Germany}

Tobacco smoke contains numerous toxic and carcinogenic substances. Some are naturally present, but many are added during manufacturing or are formed during combustion of the product. Additives are added mainly to influence the tobacco taste or for moisturizing and burning characteristics. They include any colouring agents, adhesives, plasticizers, binders, etc. that are included in the filter, the paper and in tobacco. More than 600 different additives are known, these can account for about 10 up to 25 per cent of the total weight of a cigarette. Additives transform tobacco smoke into an even more complex chemical mixture and further increase the carcinogenic and harmful effects of smoking. The presentation will demonstrate how additives are used to facilitate smoking and contribute to increased addictiveness and how flavouring agents can increase the attractiveness of tobacco products.

Tob. Prev. Cessation 2016;2(April Supplement):45

http://dx.doi.org/10.18332/tpc/62445

\section{ABSTRACT 8}

\section{Situation in France: a step forward}

Michèle Delaunay'

' French Parliament, France

The presentation will look at four key issues: The new tobacco control provisions in French Health Law (January 2016), including plain packaging and health warnings; obligation for the tobacconists to control the age of buyers, to make effective the prohibition to sales to minors; prohibition of smoking in a car with a child under 18 years; increasing the share reimbursed by French Social Security system for nicotine replacement therapies; prohibition of tobacco advertising in tobacconists shops. The weaknesses in the law and the priorities of the French Coalition against tobacco, such as the increase tobacco taxes and prices; class action for the tobacco victims; Tobacco Prevention Fund; Ensure enforcement of the law Tobacco lobbying in France: French tobacconists; Ministry of Economy; the tobacco industry... Tackling tobacco: a prerequisite to save the sustainability of our health system (health and social costs).

Tob. Prev. Cessation 2016;2(April Supplement):44 http://dx.doi.org/10.18332/tpc/62444

\section{ABSTRACT 9}

Refuting tobacco industry research on plain packaging Pascal Diethelm'

'OxyRomandie, Switzerland

In its response to the UK government consultation on plain packaging in 2014, Philip Morris International claimed that the introduction of the measure in Australia had no effect whatsoever. The company invoked studies conducted by two professors at the University of Zürich, claiming that "the experts found no evidence that 'standardised packaging' had had an effect on smoking prevalence among Australians," adding that the professors "confirmed that if there had been an effect in reality ... it would have been reflected in the data." The two studies, which were published on the UZH website as "working papers" have been criticized for their methodological 
defects. In a recent paper, published in Tobacco Prevention and Cessation, Diethelm and Farley re-analysed the data used by the two professors, and arrived at results, which refuted their findings. They found that a significant decline in smoking prevalence in Australia followed the introduction of plain packaging after adjustment for the impact of other tobacco control measures. The effect was even stronger than what the experts had predicted. What causes particular concern in the present case is the attitude of the University of Zürich: it opted for the "ostrich policy", hiding behind "academic freedom" to avoid assuming its responsibility when confronted with a clear attempt by the tobacco multinational to use the academic institution for manufacturing "science" favourable to its commercial interests.

Tob. Prev. Cessation 2016;2(April Supplement):43

http://dx.doi.org/10.18332/tpc/62443

\section{ABSTRACT 10}

\section{The ERS role on Tobacco Control Policy in Europe Christina Gratziou' \\ 'Medical School, Athens University, Greece}

The European Respiratory Society is an international medical organisation that brings together physicians, healthcare professionals, scientists and other experts working in respiratory medicine. Its aim is to alleviate suffering from respiratory diseases and promote lung health globally through science, education and advocacy. ERS has since its founding in 1990 demonstrated strong commitment to tobacco control. Through scientific assemblies, education courses, various alliances and collaboration (Framework Convention Alliance, European Chronic Disease Alliance, World Health Organisation etc.) As well as a Tobacco Control Committee (TCC) dedicated to advocacy, ERS constantly strives to promote strong and evidence-based policies to reduce the burden of tobacco related diseases. One of the main outcome of the TCC is the creation of Smokehaz, a website aimed at providing policy-makers with scientific information on the Health hazards associated with smoking. Recently, ERS created the Latin-America Working Group which aims at strengthening tobacco control activities in Spain, Portugal and Latin-American countries.

Tob. Prev. Cessation 2016;2(April Supplement):42 http://dx.doi.org/10.18332/tpc/62442

\section{ABSTRACT 11}

\section{Does 'Plain Packaging' of Tobacco matter?}

Luke Clancy1, Kate Babineaul

\section{TobaccoFree Research Institute Ireland, Ireland}

The incremental tightening of the tobacco industry's advertising capabilities led to the investment of billions of dollars into increasingly creative packaging. Today, packing remains one of the industry's sole methods of promotion and marketing. The size, shape, colour, and font on cigarette packs serve to differentiate brands and promote a certain image associated with the given cigarette package Many studies in recent years have tested the association between pack standardisation and peoples' attitudes, behaviours, and perceptions of tobacco packaging. The results show that plain packs are thought to be less attractive and associated with less positive characteristics, the health warnings are more salient, and smokers report feelings of wanting to smoke less when using standardised packs. Now we have the real life results from Australia, which show the tobacco control advances that are possible with standardised packaging. This must increase the pressure to have 'plain packaging' introduced throughout the EU. Tob. Prev. Cessation 2016;2(April Supplement):41 http://dx.doi.org/10.18332/tpc/62441

\section{ABSTRACT 12}

\section{Citizen's/Patients Role in Tobacco control}

Susanna Palkonen'

'EFA European Federation of Allergy and Airways Diseases Patients' Associations, Belgium

Health NGOs and patient groups representing people whose diseases are caused, exacerbated or worsened by smoking or exposure to tobacco smoke have a key role to play in tobacco control and advocacy, as well as a responsibility to do so. Their participation, advocacy and educational activities, such as patient education, peer support and help-line programmes are based on evidence on the impact of tobacco use. They bring the citizen's and patients' perspective, human face and impact on everyday life that tobacco continues to have in our societies. EFA, European Federation of Allergy and Airways Diseases Patients Associations and its national members take European responsibility in inserting patient perspective in tobacco control at European and national levels, in synergy with the health NGO coalition.

Tob. Prev. Cessation 2016;2(April Supplement):40 http://dx.doi.org/10.18332/tpc/62440

\section{ABSTRACT 13}

\section{Compaigning against the industry in Eurasia Region} Joshua Abrams' 


\section{'Compaign for Tobacco-Free Kids (USA)}

The Campaign for Tobacco-Free Kids and our partners have conducted a number of campaigns to expose and discredit tobacco industry interference in public policy making. The presentation will look at case studies of some of these campaigns and show how this kind of work can help defeat tobacco industry opposition to strong tobacco control laws.

Tob. Prev. Cessation 2016;2(April Supplement):39

http://dx.doi.org/10.18332/tpc/62439

\section{ABSTRACT 14}

\section{Romania- New Tobacco control law from an NGO perspective}

Florin Mihaltan', Cornel Radu Loghin², Marius Eremia', Theodor Haratau'

1 Romanian Network for Smoking Prevention, Institut of Pneumology"M.Nasta", Romanian

${ }^{2}$ European Network for Smoking and Tobacco Prevention (ENSP), Belgium

In our presentation we are evaluating the progress of the tobacco control in Romania looking form the point of view on NGO in the last 26 years. We are signalling the progressive steps towards policy and an advocacy from our perspective and the consequences of our success. The final result is reflected in the new law starting in force on March 17th, 2016, a real advance in our fight. We are identifying in the same time the new challenging problems after launching the new law. Probably the biggest gain of our fight is the extensive partnership with all the factors, our efforts to bring together in a big family as the coalition "Romania Respira" politicians, advocates, judges, economists, young's and also the new way found to encourage all: mass media, politicians, journalists, public to support us.

Tob. Prev. Cessation 2016;2(April Supplement):38 http://dx.doi.org/10.18332/tpc/62438

\section{ABSTRACT 15}

\section{Eurobarometer: an effective tool for tobacco prevention in Europe}

Nicolas Becuwe

1 TNS opinion, Belgium

The presentation will highlight the key results of the latest Eurobarometer survey about «Attitudes of Europeans towards tobacco and electronic cigarettes" which explores behaviours and attitudes of Europeans towards tobacco. This survey was conducted among more than 27,000 respondents aged 15 or more in the 28 Member States of the European Union in December 2014. The main topic will be prevalence of smoking in Europe, issues related to starting and stopping smoking, factors that influence the choice of cigarettes or e-cigarettes, exposure to tobacco smoke and advertisement and attitudes to tobacco and e-cigarette control policies.

Tob. Prev. Cessation 2016;2(April Supplement):37

http://dx.doi.org/10.18332/tpc/62437

\section{ABSTRACT 16}

Implementing the FCTC: the challenge of monitoring and accountability

Yvona Tous', Nonguebzanga Maxime Compaore ${ }^{1,2}$

' IInternational Affairs Norwegian Cancer Society, Norway

${ }^{2}$ Framework Convention Alliance, Norway

Parties to the WHO Framework Convention on Tobacco Control (FCTC) are legally bound by its provisions and are already obliged to provide detailed reports every two years on FCTC implementation. However, to date, the practical and political consequences of failure to comply are small. What is needed now is an organised system to review and follow up on the reports - an effective compliance mechanism - as has been created for many other treaties at about this stage in their development. In 2016, the Framework Convention Alliance (FCA) will advocate for the establishment of an effective FCTC compliance mechanism at the seventh session of the FCTC Conference of the Parties (COP7). The Alliance will also engage in discussions on the most meaningful and supportive role of civil society as part of this mechanism.

Tob. Prev. Cessation 2016;2(April Supplement):36

http://dx.doi.org/10.18332/tpc/62436X

\section{ABSTRACT 17}

Smoking prevention among youth: do we need educational programs?

Lucia Maria Lotrean'

' Iuliu Hatieganu University of Medicine and Pharmacy, Cluj-Napoca, Romania

This presentation focuses on smoking prevention among adolescents and young people. Highlightsinclude considerations regardingschool-based programmes,family basedinterventions, community programs, mass-media campaigns and the use of new forms of information and communication technology for smoking prevention and health promotion. It will address issues regarding development, implementation, evaluation and diffusion of smoking prevention programs for youth and 
will explore the role of educational programs within tobacco control long-term strategies.

Tob. Prev. Cessation 2016;2(April Supplement):35 http://dx.doi.org/10.18332/tpc/62435

\section{ABSTRACT 18}

The importance of using evidence-based $e$-health smoking cessation programs

Hein de Vries $^{1}$

'Maastricht University, Netherlands

eHealth programs have become very popular to help people to quit smoking. Yet, the efficacy of eHealth programs is dependent on the health communication theories used and applied in these programs. Computer tailored technology has shown to be an effective tool to help people to quit smoking. Programs with even one session can increase the success rates significantly. During this presentation I will discuss several computer tailored eHealth programs for smoking cessation that have been developed and tested at Maastricht University. I will discuss the theoretical grounding of these programs, their effects and the cost-effectiveness. Additionally I will also outline some potential innovations for eHealth programs, and will also share the results of a test comparing eHealth and mHealth.

Tob. Prev. Cessation 2016;2(April Supplement):34 http://dx.doi.org/10.18332/tpc/62434

\section{ABSTRACT 19}

\section{Taxation and Illicit Trade Protocol, "Lethal weapons"} against Tobacco Industry

Stephen Lequet $^{1}$

'Les Droits des Non-Fumeurs (DNF), France

Tobacco taxation is the most effective way to reduce smoking prevalence. Without tax increase tobacco policy, the other measures of tobacco control are weakened. While the EU Directive of 2011 on tobacco taxation is being evaluated, it is important to recall issues of this tobacco control tool. Furthermore, it is urgent to dismantle some against-truths dealt out by tobacco industry, like the supposedly postponing consumption on an illegal market which would then expanding, negating the reduction of tobacco consumption. On this important topic, the WHO Illicit Trade Protocol introduces comprehensive set of measures ranging from control supply chain, strengthening penalties and technical and legal cooperation. The EU ratification is essential.

Tob. Prev. Cessation 2016;2(April Supplement):33 http://dx.doi.org/10.18332/tpc/62433

\section{ABSTRACT 20}

\section{The role of research in tobacco control}

Filippos Filippidis'

'Imperial College London, United Kingdom

One of the main goals of public health research is to inform health-related policies. Over the past decades, tobacco control policies have been supported by robust evidence generated by research. However, decisions on tobacco control policies are not always taken based on available evidence. Other factors such as the population's attitudes, tobacco industry lobbying and socioeconomic considerations may influence policy decisions. This talk will explore the role of research in supporting evidence-based tobacco control both in the past and within the current context in Europe and beyond.

Tob. Prev. Cessation 2016;2(April Supplement):32

http://dx.doi.org/10.18332/tpc/62432

\section{ABSTRACT 21}

\section{EQUIPT: supporting European stakeholders} to make decisions about investment in evidence-based tobacco control

Profs Silvia Evers1, Hein de Vries'

'Maastricht University, The Netherlands

EQUTPT brings together expertise from multiple disciplines and aims to provide policy makers and wider stakeholders with bespoke information about the economic and wider returns that investing in evidence-based tobacco control including smoking cessation agendas can generate. Led by Health Economics Research Group (HERG) at Brunel University, London, EQUIPT is a partnership of 11 consortium members from 7 member states - Belgium, Croatia, Germany, Hungary, the Netherlands, Spain and the UK.

Tob. Prev. Cessation 2016;2(April Supplement):31

http://dx.doi.org/10.18332/tpc/62431

\section{ABSTRACT 22}

\section{The TackSHS project - a collaborative H2020 project}

Esteve Fernandez ${ }^{1,2}$

'WHO Collaborating Center for Tobacco Control

${ }^{2}$ Catalan Institute of Oncology, Spain

TackSHS is a new research project funded by the European Union's Horizon 2020 Research and Innovation Programme. It aims to improve our understanding of second-hand tobacco 
smoke and e-cigarette emissions and find ways of tackling the health burden caused by exposure to them. Within a fast changing environment, the project will try to elucidate the comprehensive impact that SHS and e-cigarette aerosols have on respiratory health of the European population and how health impacts vary according to socio-economic parameters with particular emphasis on specific vulnerable groups such as patients suffering from chronic lung diseases.

Tob. Prev. Cessation 2016;2(April Supplement):30

http://dx.doi.org/10.18332/tpc/62429

\section{ABSTRACT 23}

\section{TOB-G: Tobacco Cessation Guidelines for} High risk Populations

Panagiots Behrakis', 2, 3, 4

' European Network for Smoking and Tobacco Prevention (ENSP), Belgium

2 The American College of Chest Physicians (ACCP), USA

${ }^{3}$ Institute of Public Health, American College of Greece, Greece.

The TOB-G project is funded under the EU 3rd Health Programme which is the main instrument that the Commission uses to implement the EU Health Strategy. The project started in June 2014 and will be completed in September 2017. The project consortium consists of 5 partners from 4 European countries (Belgium, Greece, Ireland and Romania).

The TOB-G project aims to develop and implement an innovative and cost effective approach to prevent chronic diseases related to tobacco dependence by focusing on creating specialized tobacco cessation guidelines for populations of high risk including adolescents, pregnant women, adults with COPD, Cardiovascular disease and diabetes.

The specialized guidelines for high risks groups will be developed according to ENSP's evidence based and good practices in tobacco cessation. The smoking cessation guidelines contain strategies and recommendations designed to assist clinicians/ doctors in delivering and supporting effective treatments for tobacco use and dependence and will also be available within the context of an e-learning platform for European clinicians.

Overall, the TOB-G project will enhance the overall European capacity in the treatment of tobacco dependence, through offering smoking cessation tools, appropriately assessed and fitted to the specific needs of high risk groups.

Tob. Prev. Cessation 2016;2(April Supplement):29 http://dx.doi.org/10.18332/tpc/62428

\section{ABSTRACT 24}

\section{SILNE-R (Horizon2020)}

\section{Anton Kunst ${ }^{\top}$}

' University of Amsterdam, Netherlands

SILNE-R aims to evaluate how tobacco control policies can be implemented in such a way as to maximally contribute to a reduction in youth smoking in Europe. Previous evaluations basically assessed whether or not a policy was effective and cost-effective. Often, it remained unclear how some programs achieve these observed effects, or why others failed to do so. The black box remained closed. SILNE-R aims to open it. For this, we will perform in-depth analyses of the implementation and effectiveness of several policies in Finland, Germany, Belgium, Netherlands, Ireland, Italy and Portugal. We will (a) have in-depth interviews with policy makers at national and local levels, school staff, and pupils, (b) carry out the second wave of an interview survey among more than 12,000 pupils, and (c) prepare program cost inventories and produce cost-effectiveness estimates.

Based on the results, we develop and disseminate recommendations on how to implement strategies to prevent youth smoking in local settings, with due attention for program costs and for inequalities in smoking.

Tob. Prev. Cessation 2016;2(April Supplement):28

http://dx.doi.org/10.18332/tpc/62427

\section{ABSTRACT 25}

EUREST PLUS - European Regulatory Science on Tobacco: Policy implementation to reduce lung diseases - Proposal (Horizon2020)

Constantine Vardavas'

' European Network for Smoking and Tobacco Prevention (ENSP), Belgium

EUREST-PLUS, a thirteen -partner EU joint project, coordinated by ENSP (Coordinator: Constantine Vardavas), aims to monitor and evaluate the impact of the TPD at an EU level. The specific objectives of the project are:

1. To evaluate the psychosocial and behavioural impact of TPD implementation and FCTC implementation, through the creation of a longitudinal cohort of adult smokers in $6 \mathrm{EU}$ MS (Germany, Greece, Hungary, Poland, Romania, Spain) in a pre- vs. post- study design.

2. To assess support for TPD implementation through secondary dataset analyses of the 2015 Special Eurobarometer on Tobacco Survey (SETS), and through trend analyses on the merged datasets of the 2009, 2012 and2015 SETS datasets. 
3. To document changes in e-cigarette product parameters (technical design, labelling, packaging and chemical composition) following implementation of Article 20 of the TPD.

4. To enhance innovative joint research collaborations, through the pooling and comparisons across both other EU countries of the International Tobacco Control (ITC) Project, and other non-EU countries.

Tob. Prev. Cessation 2016;2(April Supplement):27

http://dx.doi.org/10.18332/tpc/62406

\section{ABSTRACT 26}

Global Bridges: building an international network for tobacco dependence treatment

Katie Kemper 1, 2

'Global Bridges, USA

${ }^{2}$ Mayo Clinic, USA

Global Bridges, hosted at Mayo Clinic, is the most diverse international network of healthcare professionals dedicated to tobacco dependence treatment. Global Bridges works with grantees and partners in all six WHO regions to educate healthcare providers on evidence-based treatment and advocate for effective tobacco policy. This session will describe accomplishments and lessons learned in the Global Bridges program over nearly six years, and opportunities to better integrate cessation support into comprehensive tobacco control.

Tob. Prev. Cessation 2016;2(April Supplement):26

http://dx.doi.org/10.18332/tpc/62426

\section{ABSTRACT 27}

\section{Smoking Cessation in COPD patients}

Carlos A. Jimenez-Ruiz'

' Smoking Cessation Service of Community of Madrid, Spain

Tobacco smoking is the main cause of COPD. Smoking cessation is the only therapeutic measure that can cure COPD and prevent this disorder from its chronic progression. Smoking cessation in COPD patients is difficult because most of these patients have specific characteristics that prevent them to quit. Recently, an ERS Task Force has developed a Consensus Document that contains recommendations for helping COPD smokers to quit.

Tob. Prev. Cessation 2016;2(April Supplement):25 http://dx.doi.org/10.18332/tpc/62425

\section{ABSTRACT 28}

\section{A brief tobacco intervention program in dental settings for developing countries}

Takashi Hanioka'

' Fukuoka Dental College, Japan

Brief intervention of smoking cessation by health professionals has been recommended worldwide. However, this practice has not been fully achieved in dental setting. The present study aimed to examine training course to pilot implementation of the WHO policy recommendations on integrating brief tobacco interventions into oral health program regarding smoking cessation for current smokers and to avoid exposure to secondhand smoke for non-smokers. We held a 1-day training course for dental professionals in Japan. The training course was organized based on the training package for primary care providers, which aims to assist countries in taking one of their first steps towards providing comprehensive tobacco dependence treatment to all tobacco users by integrating brief tobacco interventions (brief advice) into primary care. As a total, 55 oral professionals, 32 dentists and 23 dental hygienists from 19 private clinics and one university hospital, completed the training course.

Tob. Prev. Cessation 2016;2(April Supplement):24

http://dx.doi.org/10.18332/tpc/62424

\section{ABSTRACT 29}

\section{Smoking cessation services in Armenia}

Arusyak Harutyunyan', Armine Abrahamyan', Varduhi Petrosyan'

'American University of Armenia, Armenia

Eastern Europe has the highest smoking rates in Europe, yet tobacco dependence treatments are virtually unavailable in many Eastern European countries. Smoking rate among the Armenian men is one of the highest in the European region $(63 \%, 2010)$. Armenia was the first former soviet union country to accede to the WHO Framework Convention on Tobacco Control (FCTC) (2004); soon after that it adopted a national tobacco control law to ban smoking in healthcare, education, culture facilities and public transport. The country also banned tobacco advertising on TV and radio (2002) and on billboards (2006) and introduced larger (30\%) health warnings on cigarette packs (2006). One of the areas where Armenia's progress is less than satisfactory is the implementation of the FCTC Article 14. Current situation of smoking cessation services in Armenia will be presented based on the results of 
qualitative research among primary healthcare physicians and pharmaceutical market research.

Tob. Prev. Cessation 2016;2(April Supplement):23

http://dx.doi.org/10.18332/tpc/62423

\section{ABSTRACT 30}

\section{Tobacco control and cessation in Romania -} a situation analysis

Antigona Trofor', Cornel Radu-Loghin ${ }^{2}$

' University of Medicine and Pharmacy "Gr.T.Popa" Iasi, Clinic of Pulmonary Diseases Iasi, Romania

${ }^{2}$ European Network for Smoking and Tobacco Prevention (ENSP), Belgium

Romanian expertise in tobacco control and cessation is relatively young, as it has effectively started in the early ' 90 's. Ever since, smoking prevalence in the general population has decreased from $36.1 \%(48 \% \mathrm{~B} / 25 \% \mathrm{~F})$ in 2000 to $26.7 \%$ (37.4\% B/16.7\% F) in 2012, but it is still high and needs further efforts from the Romanian tobacco control community especially to address vulnerable populations and to develop tailored smoking prevention and cessation programs. Romania has ratified FCTC in 21 May 2003 and has signed it in 25 June 2004 A very recent achievement we are all proud of consists of the new law to ban smoking in public places, applied from 17.March 2016, a much stronger law than the past one we had since 2002. However, the future challenges will be to implement and ensure respect of this new law, to avoid its future amendments which already arise at the horizon, but also to introduce new regulation about taxation, illegal cigarettes traffic and e-cigarettes brands. A good point is the national Stop Smoking program that is still running since 2007 in over 50 Romanian smoking cessation centers to almost fully reimburse costs of pharmacotherapy and counselling. The constant preoccupation for education and expertise development inside the Romanian Society of Pulmonologists, in particular through its Tobaccology section established in 2007 is another plus of the Romanian tobacco control society, as well as its numerous alliances with other national and international professionals. This was fructified in various networks that have contributed to producing national smoking cessation guidelines, to creating many partnerships in tobacco control projects and in the newly successful anti-tobacco legislation.

Tob. Prev. Cessation 2016;2(April Supplement):22 http://dx.doi.org/10.18332/tpc/62408

\section{ABSTRACT 31}

Tobacco control and cessation challenges in Georgia

\section{George Bakhturidze}

'Tobacco Control Alliance, Georgia

Tobacco Control Alliance contacted the new government and requested that it fulfils its obligations under the WHO FCTC, ratified in December 2012. The Prime Minister responded adequately, and the government adopted in December 2013, its first tobacco control national Strategy and 2013-2018 action Plan. But since, nothing has really been progressed on tobacco control matters, except insufficient tax increase in September 2013 and in January 2015 (by 0.07 cents).

On the other hand, local tobacco manufacturers received around 1.5 million Euros support from the Central Budget in 2015, while the Government spent only 20000 Euros for tobacco control activities in the same year. It included monitoring on enforcement of current tobacco control law in 1400 facilities, training of 300 primary healthcare doctors on short advises technique and training for enforcement bodies.

Our group has prepared amendments to the laws on tobacco control and Government returned drafts to the $\mathrm{MoH}$ in March 2015. We organized translation of European smoking cessation guideline through ENSP support. TFK began an advocacy project in December 2015 together with us.

Tob. Prev. Cessation 2016;2(April Supplement):21

http://dx.doi.org/10.18332/tpc/62422

\section{ABSTRACT 32}

Improve the quality of brief intervention skills

Otto Stoyka'

' City Health Center of Health Department of Kyiv, Ukraine

Studied the consulting skills of physicians about patients smoking and methods of improve the quality of briefintervention skills. Conclusion: After providing training for physicians, all activities of doctors Clinical Interventions for Tobacco Use and Dependence among patients significantly increases. Doctors were twice more likely to record patients' smoking status in primary health care documents. The percentage of those who do not record patients' cigarette smoking status dropped. Also the quality of brief intervention such as advice has increased: they are more specific and contain more data about the methods and means of stopping smoking. Doctors began to treat smoking prevention more consciously and reported the usefulness and effectiveness of smoking cessation advice. The above indicates the effectiveness of efforts to improve the way doctors work in smoking prevention outpatient network. 
Tob. Prev. Cessation 2016;2(April Supplement):20 http://dx.doi.org/10.18332/tpc/62421

\section{ABSTRACT 33}

\section{Tobacco control and cessation in Eastern Europe - a} situation analysis: The Russian Perspective

Demin, Andrei', Demin Arseniy'

${ }^{1}$ Russian Public Health Association, Russia

Russia ratified WHO FCTC on 3 June 2008. On December 22, 2008 Federal law "Technical regulations on tobacco products" was adopted, probably normalizing activities of tobacco companies in view of risks posed by the Convention. Annual production capacity exceeds 700 billion cigarettes. According to GATS the highest prevalence of tobacco use in 2009 was in Russia, $39.1 \%$ of adults (43.9 million) used tobacco, smoking prevalence of $60.2 \%$ among men and $21.7 \%$ among women. In 2015, the Ministry of Health reported a $17 \%$ decline in the number of smokers since the adoption of tobacco control legislation in 2013. The law introduced comprehensive smoke free policies, picture warnings, banned point of sales materials (POSM), alas standards and order of cessation care have not been approved yet. Taxes are low and are regulated within Eurasian Economic Union agreements with a risk of remaining low. Article 5.3 implementation and signing of Protocol on Illicit Trade are among priorities.

Tob. Prev. Cessation 2016;2(April Supplement): 19 http://dx.doi.org/10.18332/tpc/62420

\section{ABSTRACT 34}

Tobacco control and cessation in Poland - a situation analysis

Krzysztof Przewozniak', Witold Zatonsk ${ }^{2}$

${ }^{1}$ Department of Cancer Epidemiology, Cancer Center and Institute of Oncology, Warsaw, Poland

${ }^{2}$ WHO Collaborating Centre, Cancer Center and Institute, Poland

In the 1980s, Poland was a country with extremely high prevalence of smoking and lung cancer mortality among men. The comprehensive tobacco control law and programs initiated in the 1990s contributed to a spectacular decrease of smoking prevalence and smoking-attributable mortality in Poland. Since the beginning of 1990, domestic cigarette sales decreased from 100 to 45 billion of cigarettes. Between 1982 and 2015, prevalence of adult smoking declined in men from $65 \%$ to $28 \%$ and from $32 \%$ to $18 \%$ in women. The fall in smoking of manufactured cigarettes is also observed in youth population.
The new challenges concern the rapid increase in smoking of hand-rolled cigarettes and in using of e-cigarettes, large discrepancies in smoking rates in educational and economic groups, rising proportion of heavy smokers and big percentage of women smoking menthol and slim cigarettes. The current tobacco control law proposal is aimed to stop these trends. Tob. Prev. Cessation 2016;2(April Supplement): 18 http://dx.doi.org/10.18332/tpc/62419

\section{ABSTRACT 35}

\section{Women and Smoking: Global Challenge}

Kunnunen Taru'

1 International Society for the Prevention of Tobacco Induced Diseases (ISPTID), USA

Global tobacco control has led to a reduction in smoking prevalence and mortality in men, while the rates among women have not followed the same declining rates or patterns. Tobacco-induced diseases, including those unique to women (reproductive complications, cervical and breast cancer) are becoming increasingly prevalent among women. Unfortunately, many tobacco control policies and cessation programs have been found to be less effective for women than men. This is alarming as disease risk for lung cancer, CVD, osteoporosis, and COPD, associated with smoking, is higher among women. Women are also more likely to be exposed to secondhand tobacco smoke and subsequent morbidity. Finally, quitting smoking appears to be harder for women than men. Current tobacco control and surveillance data come primarily from high resource countries. WHO estimates that in 2030, in low and medium resource countries, 7 out of 10 deaths will be smoking-related. While the prevalence of smoking in women is relatively low in these countries, more information is needed regarding their patterns of tobacco use uptake, and subsequent health outcomes, as theirs differ from men. Tobacco use in women is greatly influenced by social, cultural and political determinants, and needs to be conceptualized within an intersectional framework.Krzysztof Przewozniak is a Sociologist of Medicine and Methodologist of Social and Life Sciences and has over 30 years experience in the field of public health, cancer and tobacco control. As public health and tobacco control expert, he has been working or collaborating with the World Health Organization, World Bank, Centers for Disease Control and Prevention, International Union Against Cancer, International Agency for Research on Cancer, Johns Hopkins University, National Cancer Institutes in USA and Canada, US Environmental Protection Agency. He was project leader, principal investigator or collaborator in over 130 Polish 
and international research and intervention projects. He is author or co-author over 200 scientific publications. For his scientific, social and public health work, he was awarded by President of the Republic of Poland, Ministry of Health, Polish Academy of Sciences, UICC Globalink Network, and the Centers for Disease Control and Prevention in the United States.

Tob. Prev. Cessation 2016;2(April Supplement):17 http://dx.doi.org/10.18332/tpc/62407

\section{ABSTRACT 36}

\section{Caspase 3 activity in isolated fetal rat lung fibroblasts} and rat periodontal ligament fibroblasts: cigarette smoke-induced alterations

James Elliott Scott ${ }^{1}$

${ }^{1}$ University of Manitoba and Manitoba Institute for Child Health, Canada

Background Cigarette smoking is the leading cause of preventable death in the world. It has been implicated in the pathogenesis of pulmonary, oral and systemic diseases. Smoking during pregnancy is clearly a risk factor for the developing fetus and may be a major cause of infant mortality. Moreover, the oral cavity is the first site of exposure to cigarette smoke and may be a possible source for the spread of toxins to other organs of the body. Fibroblasts in general are morphologically heterogeneous connective tissue cells with diverse functions. Apoptosis or programmed cell death is a crucial process during embryogenesis and for the maintenance of homeostasis throughout life. Deregulation of apoptosis has been implicated in abnormal lung development in the fetus and disease progression in adults. Caspases, are proteases which belong to the family of cysteine aspartic acid proteases and are the key components for the downstream amplification of intra-cellular apoptotic signals. Of the 14 caspases known, caspase-3 is the key executioner of apoptosis.

Fetal rat lung fibroblasts but not PDL viability is reduced by exposure to CSE. In addition Caspase 3 activity is elevated after CSE exposure in fetal lung fibroblasts but not in PDLs. Expression of caspase 3 is induced in CSE exposed lung fibroblasts but not in PDLs. Caspase 3 was localized to the cytoplasm in both cell types.

Tob. Prev. Cessation 2016;2(April Supplement):16

http://dx.doi.org/10.18332/tpc/X62418

\section{ABSTRACT 37}

\section{Cigarette smoking and bacterial infection}

\section{David A. Scott ${ }^{1}$}

' University of Louisville, USA

Smoking cigarettes and exposure to tobacco environmental smoke increase the risk for many bacterial infections and / or exacerbates the symptoms of others. Many such infections can be life threatening. The underlying reasons for increased susceptibility are less clear. This talk will introduce examples of smoking-related bacterial diseases; and the concepts of tobacco-augmented bacterial virulence; and immune system dysregulation that may decrease resistance to microbial infections.

Tob. Prev. Cessation 2016;2(April Supplement):15 http://dx.doi.org/10.18332/tpc/62417

\section{ABSTRACT 38}

Differentiation of chronic and aggressive forms of periodontitis and of smokers and non-smokers by Fourier-transform infrared spectroscopy

Nihal Simsek Ozek', Iris Zeller', Diane E. Renaud', Panar Gümüs', Nejat Nizam', Feride Severcan', Nurcan Buduneli ${ }^{7}$, David A. Scott ${ }^{8}$

1 Ege University, School of Dentistry, Department of Periodontology, Turkey

${ }^{2}$ University of Louisville, USA

Aim: To determine if Fourier-transform infrared spectroscopy (FTIR) could distinguish chronic periodontitis (CP) and aggressive periodontitis (AgP) patients by cross-sectional salivary spectral analyses and to assess the potential confounding influence of smoking on discriminating spectral signatures.

Methods: FTIR analysis of saliva collected from patients with CP ( $\mathrm{n}=18,7$ smokers), AgP ( $\mathrm{n}=23,9$ smokers) was performed. Smoking status was confirmed by salivary cotinine analysis. Spectral band area analysis, hierarchical cluster analysis was performed.

Results: Spectral analyses indicated significantly lower lipid, phospholipid, protein, amino acid, lactic acid, nucleic acid contents in smoker than non-smoker AgP group. Amino acid, phospholipid, lactic acid contents were significantly lower in smoker than non-smoker CP group. Thiocyanate levels successfully differentiated smokers from non-smokers, irrespective of periodontal status. Cluster analysis to discriminate smokers from non-smokers and CP from AgP was highly promising. Conclusions: FTIR can be employed to discriminate smokers from non-smokers and CP from AgP. Tob. Prev. Cessation 2016;2(April Supplement):14 http://dx.doi.org/10.18332/tpc/62416 


\section{ABSTRACT 39}

Lung cancer and smoking among women - a new challenge in Europe

Witold Zatonski', Krzysztof Przewozniak²

${ }^{1}$ Health Promotion Foundation, Poland

2 Polish Civil Coalition Tobacco or Health, Poland

This paper presents the picture of lung cancer mortality among women in the European Union in relation to patterns and trends in women smoking as a rising public health problem. Based on WHO mortality statistics and international data on smoking prevalence, this analysis shows that lung cancer mortality among European women has been increasing for many years, and in the most of countries is already or will be in the next decade the leading cause of cancer deaths. The increase in lung cancer mortality is caused by a persistent epidemic of tobacco smoking in women and strongly associated with age patterns of smoking behaviours. Polish data also show on the consistency of lung cancer epidemic in men and women population, although there is a time lag in this epidemic in female population. Smoking prevention and cessation should remain a priority of cancer primary prevention and health promotion in women.

Tob. Prev. Cessation 2016;2(April Supplement):13

http://dx.doi.org/10.18332/tpc/62415

\section{ABSTRACT 40}

Cytisine in the treatment of tobacco dependence: safety, efficacy, market in Eastern Europe

Aleksandra Herbe', Witold Zatoski ${ }^{1}$

1 Health Promotion Foundation, Poland

Cytisine is a generic partial agonist of nicotine receptors a4ß2 that has been available in Eastern Europe since 1960s. Through decades of observations and clinical studies, with much research conducted in Poland, cytisine has been shown to be safe and highly cost-effective medication in treatment for tobacco dependence, with a profile potentially more favourable than that of varenicline. Poland remains among few European countries allowing for sales of cytisine. Currently, cytisinebased treatment is available over-the counter in Poland, where it is the dominant form of cessation pharmacotherapy. This presentation will outline the current state of knowledge on cytisine, as well as discuss the observations on sales and use of cytisine in treatment of smoking cessation in Poland. A case will be made for cytisine emerging as an 'aspirin' in smoking cessation, and an important element of future tobacco control worldwide.
Tob. Prev. Cessation 2016;2(April Supplement):12

http://dx.doi.org/10.18332/tpc/62414

ABSTRACT 41

\section{Availability, Affordability, and Prices of Smoking Cessation Products in 9 Countries: Preliminary Findings}

Arusyak Harutyunyan

' Center for Health Services Research and Development (CHSR), Armenia

Numerous effective medications are available for tobacco dependence treatment, and clinicians should encourage their use by all patients attempting to quit smoking. According to the FCTC Article 14 Guideline "Tobacco cessation medications should be made available to tobacco users wanting to quit and where possible be provided free or at an affordable cost". Online survey was conducted among 9 countries (Armenia, Bangladesh, Brazil, India, Jordan, Nigeria, Spain, Turkey, Vietnam) to determine availability, affordability, and prices of smoking cessation drugs in countries that are Global Bridges grantees and to make comparisons across them. Preliminary findings of the online survey will be summarized during the presentation.

Tob. Prev. Cessation 2016;2(April Supplement):11 http://dx.doi.org/10.18332/tpc/62413

\section{ABSTRACT 42}

TiTAN Crete (Tobacco Treatment Training Network in Crete). Designing Smoking Cessation intervention in Primary Care Practice: Preliminary data

Charis Girvalaki', Sophia Papadakis², Constantine Vardavas', Andrew Pipe ${ }^{2}$, Christos Lionis'

'Clinic of Social and Family Medicine, Department of Medicine, University of Crete, Greece

${ }^{2}$ University of Ottawa, Canada

Aims and Objectives: We report on baseline tobacco treatment rates and changes in evidence-based tobacco treatment knowledge among General Practitioners (GPs), who participated in the TiTAN Crete training-based intervention. Methods: A pre-post control group evaluation was conducted. GPs $(n=15)$ completed a survey prior to and immediately following the training session and a cross sectional patient sample of patients $(n=540)$ was sampled pre-post intervention.

Results: Tobacco use prevalence was $38 \%$. The majority (64.1\%) of tobacco users reported readiness to quit smoking in the next 6 -months and $63.4 \%$ reported not having made a quit 
attempt in the past year. GPs tobacco treatment knowledge was limited at baseline. Significant increases in knowledge were documented in $6 / 13$ of the knowledge domains. A followup survey to examine changes in rates of tobacco treatment delivery among GPs is underway.

Conclusions: The TiTAN Crete training program was effective in increasing providers tobacco related knowledge.

Tob. Prev. Cessation 2016;2(April Supplement):10

http://dx.doi.org/10.18332/tpc/62412

\section{ABSTRACT 43}

\section{Helping people stop smoking in workplaces: an} alternative path

Joseph Osman'

'OFT Conseil, France

About 16 million people smoke in France. Those who have decided voluntarily to stop, or those who need to stop for medical reasons are looking for help in one of the 800 smoking cessation medical centres. They represent a tiny part of the active smokers.

An alternative way to reach and help smokers who do not want to stop or do not even consider stopping is to follow them through specific programs in their workplaces.

Initiated by Prof Bertrand Dautzenberg 10 years ago "OFT" and then "OFT Conseil" have been developing different plans to address those smokers where they are working and encourage them to stop.

The fact is that many medium and large size companies want their staff to stop smoking to keep people in good health, to protect the image of the company or for any other reason. We are generally commissioned by the Occupational Health Doctors or by Human Resources Managers to intervene. Each year OFT tobaccologists are working in some 75 to 100 companies.

OFT and later OFT Conseil have developed a 3 steps programme especially addressed to workplaces. The 3 steps are complementary although not mandatory: 1 . Group meeting with smokers to raise awareness of well-being after stopping 2. Individual interview to provide a diagnosis and build up an individual cessation programme 3. A smoking cessation protocol based on 6 to 10 consultations over 6 to 8 months

This plan is financed by the companies and followed only by graduated tobaccologists (faculty of medicine).

The success rate in average is more or less $50 \%$ to $60 \%$ in the best cases (smokers who follow regularly/fully the programme).
Tob. Prev. Cessation 2016;2(April Supplement):9 http://dx.doi.org/10.18332/tpc/62411

\section{ABSTRACT 44}

\section{The Hookah: Not Benign and Not Cultural}

Barry A. Finegan'

' University of Alberta, Canada

Exposure to hookah has burgeoned among youth with 40\% responding to "ever used?" in a recent UK sample. Hookah use has been touted as safe especially if 'herbal' shisha is smoked and defended as a 'cultural' practice when banning hookah use in public spaces is proposed. Our data convincingly demonstrates that both these assertions are baseless. 'Herbal' shisha products tested contained toxic trace metals and PAHs levels equivalent to, or in excess of, that found in cigarettes. Their mainstream and side stream smoke emissions contained carcinogens equivalent to, or in excess of, those of tobacco products. The content of the air in the water pipe cafés tested where 'herbal' products were smoked contained potentially dangerous micro-particle and CO levels. Our qualitative research strongly suggests that peer influence, availability of flavoured products and facile access to hookahs in cafés are the major factors in initiation. Ethno-cultural issues play only a minor role.

Tob. Prev. Cessation 2016;2(April Supplement):8

http://dx.doi.org/10.18332/tpc/62410

\section{ABSTRACT 45}

\section{Tobaccofree Sports Stadia: European Policies and Guidance}

Matthew Philpott ${ }^{\prime}$

' European Healthy Stadia Network, UK

Over 20 million people - including many children and young people - attend professional football matches across Europe each week, with many more attending other professional and amateur sports fixtures. The vast majority of European sports venues take place in semi-enclosed structures with roofed stands, and yet there are still very low levels of smokefree and tobaccofree policies in operation. Building on research findings that only 10 out of UEFA's 54 European countries have football stadia that operate tobaccofree policies, Healthy Stadia has worked with UEFA and World Heart Federation to develop new guidance materials for clubs, multi-purpose stadium operators and governing bodies of sport to assist in the development and enforcement of strong tobacco control policies. This presentation will give a benchmark of current 
policies in place, support offered through the new guidance materials, and advice on how sports organisations can go beyond national tobacco control legislation to implement tobaccofree policies.

Tob. Prev. Cessation 2016;2(April Supplement):7 http://dx.doi.org/10.18332/tpc/62409 